ARTÍCULO

\title{
Actitudes hacia la discapacidad en una universidad mexicana
}

\author{
Araceli Arellano Torres' D \\ Martha Leticia Gaeta González" (D) \\ Feli Peralta López' (D) \\ Judith Cavazos Arroyo"
}

\section{RESUMEN}

Las actitudes negativas hacia las personas con discapacidad actúan como barreras invisibles, impidiendo la inclusión y participación de dicha población en ámbitos educativos, especialmente en instituciones de educación superior. La investigación sobre actitudes, en México, sigue siendo un campo relativamente novedoso de conocimiento. Se presentan en este artículo dos estudios que analizan la presencia de prejuicios y actitudes hacia la discapacidad, en el entorno universitario. Se usan dos instrumentos con dos muestras diferentes $(n=611$ y $n=308)$ y se presentan los datos sobre las actitudes y prejuicios y su relación con diferentes variables moderadoras como edad, sexo o contacto previo con personas con discapacidad. Los participantes presentan actitudes positivas y escasos prejuicios hacia la discapacidad. No se reportan diferencias significativas en función de variables sociodemográficas ni de contacto.

PALABRAS CLAVE

discapacidad; inclusión; universidad; actitudes; prejuicios. 


\section{ATTITUDES TOWARD DISABILITY IN A MEXICAN UNIVERSITY}

\section{ABSTRACT}

Negative attitudes towards people with disabilities act as invisible barriers to the inclusion and participation of this population in educational settings, especially in higher education institutions. The research on attitudes, in Mexico, remains a relatively new field of knowledge. This paper presents two studies that analyse prejudices and attitudes toward disability of undergraduate students in a university environment. Two instruments with two different samples $(\mathrm{n}=611$ and $\mathrm{n}=308)$ are used. We report data on attitudes and prejudices and their relationship with different moderating variables such as age, sex or prior contact with people with disabilities. Participants show positive attitudes and few prejudices toward disabilities. No significant differences are reported according to sociodemographic or contact variables.

KEYWORDS

disability; inclusion; university; attitudes; prejudices.

\section{ATITUDES EM RELAÇÃO À DEFICIÊNCIA EM UMA UNIVERSIDADE MEXICANA}

RESUMO

Atitudes negativas atuam como barreiras invisíveis para a inclusão de pessoas com deficiência em ambientes educacionais, especialmente de ensino superior. A pesquisa sobre atitudes no México continua sendo um campo relativamente novo de conhecimento. Este artigo apresenta dois estudos que analisam preconceitos e atitudes em relação à deficiência de estudantes de graduação em uma universidade. Dois instrumentos com duas amostras (n=611 e n=308) são usados. Relatamos dados sobre atitudes e preconceitos e sua relação com diferentes variáveis moderadoras, tais como idade, sexo ou contato prévio com pessoas com deficiência. Os participantes mostram atitudes positivas e poucos preconceitos em relação à deficiência. Nenhuma diferença significativa é relatada de acordo com variáveis sociodemográficas ou de contato.

PALAVRAS-CHAVE

deficiência; inclusão; universidade; atitudes; preconceitos. 


\section{INTRODUCCIÓN}

Garantizar el desarrollo personal, educativo, social y laboral de las personas con discapacidad es uno de los grandes retos a los que la población mexicana se enfrenta. No en vano, y no solamente en México, las personas con discapacidad son uno de los colectivos más estigmatizados y discriminados en todos los ámbitos de su vida (Blanco,2006; Morin et al.,2013; Verdugo y Schalock,2013). Desde hace años, los datos que la Encuesta nacional sobre discriminación en México (INEGI, 2005, 2010, 2017) arroja son mayoritariamente negativos: las personas con discapacidad no cuentan con las mismas oportunidades para su plena participación en la sociedad, menos aun cuando además se añaden otras variables como género o etnicidad, lo cual es conocido como discriminación múltiple.

Si bien son muchas las barreras aún presentes para conseguir la inclusión en todas las áreas mencionadas, también es cierto que se han producido avances significativos en el logro de los derechos de esta población. En el marco que estable la Ley General para la Inclusión de las Personas con Discapacidad ${ }^{1}$ (México, 2011), en México, se otorga prioridad a las actuaciones en el ámbito educativo, por considerarse que la exclusión educativa es, de alguna manera, origen de otros muchos procesos de exclusión (Davis y Watson, 2001).

Teniendo en cuenta esta realidad, es prioritario indagar acerca de la situación de las personas con discapacidad en los ámbitos educativos, atendiendo a la compleja red de variables que promueven, o dificultan, la igualdad de oportunidades y el acceso a la educación de calidad en todas las etapas. En ese sentido, desde diferentes organismos e instituciones se aborda cómo transcurrir hacia la escuela inclusiva en América Latina, tomando como base el modelo social, aceptado por la comunidad científica como el enfoque más adecuado para la interpretación de la discapacidad (ver, por ejemplo, publicaciones de la UNESCO, 2004, 2005).

Este modelo, que también constituye el sustento teórico de nuestro trabajo, entiende que las dificultades de la persona para el logro de una vida de calidad no dependen exclusivamente de sus limitaciones individuales sino, sobre todo, de la interacción de las mismas con las condiciones de su contexto. Esto es, son las barreras presentes en el entorno las que obstaculizan en mayor medida la participación y no tanto las características del sujeto (Palacios y Bariffi, 2007; Verdugo, 2001). Por otra parte, y en cuanto a factores relacionados con el entorno, se ha diferenciado entre elementos que tienen que ver con barreras físicas y elementos que tienen que ver con barreras psicológicas. Estas últimas, referidas, entre otras cosas, a las actitudes hacia la discapacidad, suponen con frecuencia importantes obstáculos que dificultan la presencia, aprendizaje y participación de todos los alumnos, en igualdad de condiciones (Polo y López, 2006).

A nivel internacional, buena parte de los esfuerzos realizados hasta el momento, con el objetivo de eliminar barreras presentes, se centran en la etapa de la educación

1 Entendiendo por persona con discapacidad aquella que, por razón congénita o adquirida presenta una o más deficiencias de carácter físico, mental, intelectual o sensorial, ya sea permanente o temporal y que al interactuar con las barreras que le impone el entorno social, pueda impedir su inclusión plena y efectiva, en igualdad de condiciones con los demás (México, 2011, p. 4). 
básica, caracterizada por mayores índices de inclusión de alumnado con discapacidad, aunque en el caso de América Latina aún queda lejos la universalización de la educación primaria (Blanco, 2006). Se prioriza la formación de los maestros, se implantan medidas de atención a la diversidad y, cada vez con más éxito, se asume un modelo educativo inclusivo dirigido a todos los alumnos, sin excepción, independientemente de su características o necesidades. Según revisiones como las de García (2011), Romero y García (2013), o Ezcurra (2003), los principales avances al respecto en el sistema educativo mexicano son: el incremento de alumnos con discapacidad atendidos en escuelas regulares, actitudes cada vez más positivas de todos los agentes educativos (familias, profesores, compañeros), y la mejora en los procesos de formación de los educadores, especialmente de maestros regulares en medidas de atención a la diversidad.

Sin embargo, existe todavía una brecha importante entre la atención que reciben los alumnos con discapacidad en etapas inferiores, obligatorias, y en niveles educativos superiores, no obligatorios. Es decir, las instituciones de educación superior, no solamente en México, están reaccionando con mayor lentitud a la hora de adoptar un modelo inclusivo (Alcántara y Navarrete, 2014; Cruz y Casillas, 2017). Las universidades son señaladas, con frecuencia, como instituciones especialmente resistentes al cambio, con barreras importantes para la plena inclusión (Tapia y Manosalva, 2012).

Centrándonos en México, no existen por el momento, datos sistemáticos acerca de la presencia de estudiantes con discapacidad en las universidades, algo que limita la creación de condiciones favorables y programas que favorezcan el desarrollo educativo de dichos alumnos. En un estudio reciente, Cruz y Casillas (2017) examinan un total de 53 universidades mexicanas con el objetivo de analizar la presencia de elementos y acciones que promuevan la inclusión de personas con discapacidad (políticas institucionales, programas específicos, servicios de apoyo, etc.). Solamente, en 12 de dichas universidades se reportaron alguno(s) de estos elementos, siendo este dato - además - insuficiente para asegurar que son universidades inclusivas y accesibles.

En ese sentido, y en vistas a la escasa presencia de la discapacidad en las universidades, cabría analizar variables relacionadas con la organización, las políticas y las estructuras propias de estas instituciones ¿Por qué la educación superior no está aún preparada para acoger en igualdad de condiciones a las personas con discapacidad? ¿Por qué las universidades siguen generando desigualdades y discriminaciones, aun cuando esto va en contra de su propia naturaleza como espacios de formación? Por otro lado, cabe cuestionarse las actitudes hacia las personas con discapacidad que forman parte también de la cultura universitaria. Si bien son estos aspectos menos perceptibles directamente, ya se ha mencionado su relevancia como barreras poderosas a la inclusión. En el marco anterior, se presentan en este artículo dos estudios relacionados con esta última cuestión: el papel de las actitudes hacia la discapacidad en un entorno universitario.

\section{ACTITUDES HACIA LA DISCAPACIDAD}

En el estudio de las causas de la discriminación o segregación, especialmente desde el modelo social mencionado, se señalan frecuentemente las actitudes ne- 
gativas hacia las personas con discapacidad como importantes barreras invisibles (Goreczny et al., 2011; Martínez y Bilbao, 2011; Scior y Furnham, 2011; Werner et al. 2012). En este trabajo, partimos de la definición clásica de actitudes como aquellos procesos mentales que determinan una serie de respuestas, relativamente estables, con relación a un objeto (Allport, 1954; Triandis, 1971). Abordaremos, además de las actitudes entendidas de modo genérico, los prejuicios definidos como un tipo de actitud negativa y hostil hacia las personas con discapacidad por el hecho de pertenecer a ese colectivo.

Las actitudes han sido objeto de análisis durante años, no solamente en el ámbito educativo. Buena parte de la investigación a este respecto, se ha centrado en examinar las actitudes, tanto de la población general como de colectivos específicos, hacia las personas con discapacidad (para una revisión, ver el trabajo de Scior, 2011). Se constata que las actitudes hacia este grupo siguen siendo mayoritariamente negativas, a pesar de cierta evolución positiva durante los últimos años (Verdugo y Schalock, 2013). La investigación indica que las actitudes varían de acuerdo con una serie de variables moderadoras tales como el sexo, la edad o las experiencias de contacto con personas con discapacidad. En ese sentido, parecen existir ciertas tendencias: las mujeres muestran actitudes más positivas que los hombres (Dachez, Ndobo y Ameline, 2015; Goreczny et al., 2011), las personas que tienen contacto con personas con discapacidad muestran actitudes más positivas (Barr y Bracchitta, 2015; Cairns y McClatchey, 2013; García y Hernández, 2011; Morin et al., 2013; Ouellette-Kuntz et al., 2010; Pettigrew y Tropp, 2006; Seo y Chen, 2009) y las actitudes hacia personas con discapacidad física son más positivas que hacia aquellas con discapacidad intelectual (Barr y Bracchitta, 2015; Findler, Vilchinsky y Werner, 2007; Moreno et al.,2006). No obstante, existen también hallazgos contrarios a estas conclusiones. En el caso de García y Hernández (2011) señalan que las actitudes hacia las personas con discapacidad intelectual son más positivas que hacia aquellas con discapacidades físicas o sensoriales y que el contacto, cuanto menos frecuente, más relacionado está con actitudes positivas. También existen resultados contradictorios respecto a las actitudes de grupos de diferente edad: estudios como los de Goreczny et al. (2011), Morin et al. (2013) o García y Hernández (2011), encuentran que los jóvenes mantienen actitudes más positivas que las personas mayores, mientras que Harper y Peterson (2001) o Ten-Klooster et al. (2009) aportan lo contrario.

En el caso de México, son escasos los estudios que analicen las actitudes hacia este grupo de población y prácticamente inexistentes en entornos universitarios. Encontramos trabajos como los de García y Hernández (2011) que reportan que aquellas personas con un nivel educativo alto, jóvenes (13-30 años), procedentes de zona urbana, que no profesan ninguna religión y que han mantenido contacto esporádico con personas con discapacidad, tienen actitudes más positivas que aquellos del ámbito rural, con menor formación, de mayor edad, pertenecientes a una religión determinada, sin contacto previo con esta realidad. Por su parte, Barradas et al. (2014), con una muestra de académicos, en el estado de Veracruz, analiza las actitudes hacia la discapacidad motriz. Su conclusión es que las actitudes son mayoritariamente positivas aunque existen ciertos aspectos de mejora (por ejemplo, la disposición de los sujetos a interactuar con personas con discapacidad). 
Estos hallazgos coinciden en general con los de Cruz (2016), con miembros de la comunidad universitaria (estudiantes, docentes y personal administrativo), también de Veracruz, que apuntan a actitudes favorables hacia las personas con discapacidad, pero que plantean una discriminación latente al no contar con los medios mínimos necesarios para el acceso de este colectivo a la universidad. Estos son ejemplos de estudios sobre esta temática, pero cuyas conclusiones están lejos de ser definitivas.

$\mathrm{El}$ análisis de las actitudes es tarea compleja dado que hacen referencia a una realidad multidimensional y, con frecuencia, interpretada desde posiciones muy variadas. Quizá por eso, tanto a nivel internacional como en el caso de México, los datos respecto a los factores que están relacionados con las actitudes son, en gran parte, todavía confusos. En el caso de los prejuicios, diversos investigadores han planteado la posibilidad de que existan dos tipos de actitudes negativas: clásicas (overt attitudes - prejuicios explícitos, que con el tiempo reflejan una perspectiva políticamente incorrecta) y modernas (covert attitudes - prejuicios implícitos, que de una forma más sutil reflejan rechazo) (Akrami, Ekehammar y Araya, 2000; Akrami et al. 2006). Estos últimos estarían caracterizados por tres componentes: negar la discriminación, oposición a las demandas del grupo de discriminado y resentimiento hacia el "trato de favor" que podría recibir dicho grupo. Esta distinción, que además se ha identificado en contextos culturales diversos, explicaría en parte la desaparición de ciertas actitudes negativas que, sin embargo, al surgir otras nuevas no consigue frenar la discriminación (Akrami et al., 2006).

En el marco anterior, se presentan en este trabajo dos estudios que permitan ampliar los datos disponibles sobre actitudes hacia las personas con discapacidad en México, en un contexto universitario: uno de ellos centrado en los prejuicios (Estudio 1), basándonos en la teoría de prejuicios explícitos e implícitos, y otro centrado en las actitudes hacia la discapacidad (Estudio 2) entendidas de un modo más genérico.

\section{MÉTODO}

Ambos estudios se llevaron a cabo desde un enfoque cuantitativo, mediante un diseño exploratorio de corte transversal, en el que se aplicaron instrumentos estandarizados de autoreporte a estudiantes de maestría en el área de pedagogía, en la ciudad de Puebla (México). Los objetivos concretos de esta investigación son:

- valorar los prejuicios y actitudes hacia las personas con discapacidad en dos grupos de universitarios;

- comprobar la adecuación de dos instrumentos a la población de estudio; y

- examinar la relación que guardan los prejuicios y las actitudes con determinadas variables sociodemográficas y personales.

Los datos del Estudio 1 fueron recogidos durante mayo de 2014, mientras que el Estudio 2 se llevó a cabo durante mayo de 2016. En ambos casos, los datos se analizaron con el paquete estadístico SPSS versión 20.0. Se realizó un estudio de las principales características psicométricas de los instrumentos, así como una exploración de la normalidad de los datos y la homogeneidad de las varianzas, un análisis descriptivo de las distintas variables (análisis de frecuencias, medias y desviación típica) y una comparación de las mismas según características demográficas. 


\section{ESTUDIO 1}

\section{PARTICIPANTES}

La muestra constó de 611 estudiantes (Tabla 1).

Tabla 1 - Datos sobre la muestra de estudio $(n=611)$.

\begin{tabular}{l|c|c|c}
\hline Variables & Datos sociodemográficos & $\mathrm{Fr}$ & $\%$ \\
\hline \multirow{3}{*}{ Sexo } & Hombre & 97 & 15,9 \\
\cline { 2 - 4 } & Mujer & 514 & 84,1 \\
\hline \multirow{4}{*}{ Edad } & $20-30$ & 363 & 59,4 \\
\cline { 2 - 4 } & $31-40$ & 179 & 28,8 \\
\cline { 2 - 4 } & $41-50$ & 52 & 8,5 \\
\hline \multirow{3}{*}{ Contexto laboral } & $51-60$ & 17 & 2,7 \\
\hline \multirow{4}{*}{$\begin{array}{l}\text { Años de experiencia } \\
\text { profesional }\end{array}$} & Urbano & 366 & 59,9 \\
\cline { 2 - 4 } & Semiurbano & 126 & 20,6 \\
\cline { 2 - 4 } & Rural & 119 & 19,4 \\
\cline { 2 - 4 } & $0-5$ & 334 & 54,66 \\
\cline { 2 - 4 } & $6-10$ & 94 & 15,38 \\
\cline { 2 - 4 } & $11-15$ & 50 & 8,18 \\
\cline { 2 - 4 } & $16-20$ & 14 & 2,29 \\
\cline { 2 - 4 } & $21-25$ & 103 & 1,14 \\
\cline { 2 - 4 } & $26-30$ & 1,3 \\
\hline
\end{tabular}

Fr: frecuencia.

Fuente: Base de datos de la investigación.

Elaboración de las autoras.

\section{INSTRUMENTO}

Los datos se recogieron a través de un cuestionario formado por tres secciones. La sección 1 incluye variables sociodemográficas y personales que han mostrado cierta relación con las actitudes hacia las personas con discapacidad, en investigaciones previas. La sección 2 recoge el contacto con personas con discapacidad, dado su papel potencial en la explicación y predicción de las actitudes. La sección 3 está formada por la escala Modern and Classical Attitudes Scales Toward People with Intellectual Disabilities (Akrami et al., 2006). Contiene 19 ítems que se responden según una escala de tipo Likert ( 1 = Fuertemente en desacuerdo a $5=$ Fuertemente de acuerdo), de las cuales 8 reflejan prejuicios explícitos y 11 prejuicios implícitos. Los datos acerca de la fiabilidad y consistencia interna de la escala, para esta muestra, aportan un Alfa de Cronbach del conjunto de ítems de 0,711 (19 ítems). No obstante, si analizamos según factores, prejuicios clásicos o explícitos y 
modernos o implícitos, muestran valores menores, no aceptables. Por lo tanto, se ha utilizado la escala en su conjunto para reflejar la variable "prejuicios", aunque se tendrá en cuenta la presencia de los dos factores en la interpretación de resultados.

\section{RESULTADOS}

En cuanto al contacto previo con la discapacidad, del total de participantes ( $\mathrm{n}=611$ ), la mayoría afirmó conocer o haber conocido alguna persona con discapacidad $(n=404)$. De manera más específica, los participantes señalaron tener familiares con discapacidad $(n=113)$, contar con experiencia profesional con personas con discapacidad $(n=155)$, tener experiencia de voluntariado con personas con discapacidad $(n=60)$, tener/haber tenido compañeros de escuela o trabajo, amigos o conocidos $(n=59)$ y algunos señalaron haber tenido contacto con personas con discapacidad pero no especificaron su parentesco con ellas $(n=17)$. Respecto al tipo de discapacidad con la que han tenido contacto, se mencionaron trastornos asociados a todos los tipos de dificultades: discapacidad auditiva, discapacidad intelectual, discapacidad física y discapacidad visual (algunos de ellos: trastorno del espectro autista, déficit de atención e hiperactividad, dislexia, distrofia muscular, epilepsia, hidrocefalia, parálisis cerebral, problemas de lenguaje, síndrome de Down y síndrome de West).

En cuanto a las respuestas de la escala de Akrami et al.(2006), la puntuación promedio de los participantes es de 2,30 $(\mathrm{DT}=0,39)$ (siendo las puntuaciones más altas indicativo de mayores prejuicios). La mayoría de los ítems obtienen valores por debajo de 3. Únicamente, encontramos un ítem en valores más altos: las personas con discapacidad intelectual se están volviendo muy exigentes en su lucha por la igualdad de derechos (valor medio $=3,23 ; \mathrm{DT}=1,08$ ). Los ítems con puntuaciones más bajas son: como el resto, las personas con discapacidad intelectual tienen objetivos y metas en su vida (valor medio $=1,88 ; \mathrm{DT}=0,84$ ) y es justo que las personas con discapacidad intelectual reciban apoyo especial de la sociedad para encontrar puestos de trabajo adecuados (valor medio $=1,97 ; \mathrm{DT}=0,89$ ).

En cuanto a la incidencia que tienen las variables sociodemográficas y de contacto sobre las actitudes, dada la no normalidad de los datos, los análisis no paramétricos realizados ( $U$ de Mann-Whitney para dos muestras independientes y Kruskal-Wallis para muestras múltiples) indicaron que no hay diferencias significativas en la escala global. Así lo señalan los análisis para comparar grupos en función del sexo $(\mathrm{z}=0,887 ; \mathrm{p}=0,375)$, los años de experiencia $\left(\chi^{2}=2,44 ; \mathrm{p}=0,486\right)$, el tipo de contexto laboral $\left(\chi^{2}=0,105 ; p=0,949\right)$, y la edad $\left(\chi^{2}=2,97 ; p=0,226\right)$. Tampoco existen diferencias significativas con relación al contacto previo personal $(\mathrm{z}=1.477 ; \mathrm{p}=0,140)$, profesional ( $\mathrm{z}=1.294 ; \mathrm{p}=0,196)$, o como voluntarios con personas con discapacidad $(\mathrm{z}=0,829 ; \mathrm{p}=0,407)$.

Sí se encontraron diferencias significativas en ítems concretos, según los grupos, como se describe a continuación. En cuanto al sexo, se observaron diferencias en los ítems siguientes: es justo que las personas con discapacidad intelectual a veces reciban apoyo especial de la sociedad para encontrar puestos de trabajo adecuados $(\mathrm{z}=2,48 ; \mathrm{p}=0,013)$; las personas con discapacidad intelectual reciben muy poca atención en los medios $(\mathrm{z}=2,72 ; \mathrm{p}=0,006)$; sería imprudente que una persona 
sin discapacidad intelectual se casara con una persona con discapacidad intelectual $(z=2,47 ; p=0,013)$. En todos los casos, las mujeres apuntan actitudes más positivas.

En lo que refiere a la edad, se encontraron diferencias significativas en los ítems siguientes: como el resto de las personas, las personas con discapacidad intelectual tienen objetivos y metas en su vida $(\mathrm{z}=2,19 ; \mathrm{p}=0,029)$; sería imprudente que una persona sin discapacidad intelectual se casara con una persona con discapacidad intelectual $(\mathrm{z}=2,26 ; \mathrm{p}=0,024)$. En ambos casos, las personas con más de 30 años de edad manifiestan actitudes más negativas. En cuanto a los años de experiencia laboral, se encontraron diferencias significativas en los ítems siguientes: como el resto de personas, las personas con discapacidad intelectual tienen objetivos y metas en su vida $\left(\chi^{2}=8,57 ; \mathrm{p}=0,036\right)$; sería imprudente que una persona sin discapacidad intelectual se casara con una persona con discapacidad intelectual $\left(X^{2}=9,82 ; p=0,020\right)$; las personas con discapacidad intelectual tienen menos fuerza de carácter que las personas sin discapacidad intelectual $\left(\chi^{2}=11,44 ; p=0,010\right)$.

En todos los casos, las personas con más de 20 años en el trabajo apuntan más prejuicios. Respecto al contexto de los participantes (urbano, rural, semiurbano) se observaron diferencias significativas en el ítem: las personas con discapacidad intelectual tienen menos fuerza de carácter que las personas $\sin$ discapacidad $\left(\chi^{2}=7,72\right.$; $p=0,021)$. Las personas que trabajan en un contexto urbano señalan mayores prejuicios que las personas de un contexto semiurbano $(z=2,74 ; p=0,006)$. Finalmente, los participantes que no conocen a personas con discapacidad refieren mayores prejuicios, reflejados en los ítems: la mayoría de las personas con discapacidad intelectual carecen de las cualidades que cualquier miembro de la comunidad debe tener $(\mathrm{z}=2,76 ; \mathrm{p}=0,006) ;$ las personas con discapacidad intelectual deberían vivir en lugares protegidos debido a los peligros presentes en la sociedad $(\mathrm{z}=2,23 ; \mathrm{p}=0,026)$.

\section{ESTUDIO 2}

\section{PARTICIPANTES}

La muestra constó de 308 estudiantes de la misma universidad que los participantes del Estudio 1 (Tabla 2).

Tabla 2 - Datos sobre la muestra de estudio $2(\mathrm{n}=308)$.

\begin{tabular}{l|c|c|c}
\hline Variables & Datos sociodemográficos & Fr & $\%$ \\
\hline \multirow{2}{*}{ Sexo } & Hombre & 97 & 31,5 \\
\cline { 2 - 4 } & Mujer & 211 & 68,5 \\
\hline \multirow{3}{*}{ Edad } & $20-30$ & 189 & 61,4 \\
\cline { 2 - 4 } & $31-40$ & 64 & 20,8 \\
\cline { 2 - 4 } & $41-50$ & 42 & 13,6 \\
\cline { 2 - 4 } & $51-60$ & 13 & 4,2 \\
\hline
\end{tabular}

Fr: frecuencia.

Fuente: Base de datos de la investigación.

Elaboración de las autoras. 


\section{INSTRUMENTO}

El cuestionario, también en este caso, está formado por tres secciones: sección 1 , sobre variables demográficas, sección 2 , sobre contacto con personas con discapacidad (comunes con el Estudio 1) y sección 3, compuesta por la Escala Actitudes hacia las personas con discapacidad, Forma $G$, de Verdugo, Arias y Jenaro (1994). Consta de 37 ítems con respuesta de tipo Likert ( $1=$ Muy de acuerdo con $6=$ Muy en desacuerdo), distribuidos en cinco factores:

1. Valoración de capacidades y limitaciones;

2. Reconocimiento/Negación de derechos;

3. Implicación personal;

4. Calificación genérica; $y$

5. Asunción de roles.

Se examinó la fiabilidad del instrumento, encontrando los valores Alfa de Cronbach que se muestran a continuación (Tabla 3).

Tabla 3 - Índices de fiabilidad de la

Escala Actitudes hacia las personas con discapacidad.

\begin{tabular}{l|c|c}
\hline Factor & Ítems & $\alpha$ \\
\hline 1 & 10 & 0,73 \\
\hline 2 & 11 & 0,81 \\
\hline 3 & 7 & 0,74 \\
\hline 4 & 5 & 0,52 \\
\hline 5 & 4 & 0,72 \\
\hline Total & 37 & 0,90 \\
\hline
\end{tabular}

Fuente: Base de datos de la investigación.

Elaboración de las autoras.

\section{RESULTADOS}

Del total de participantes $(n=308)$, aquellos que afirmaron haber tenido contacto con personas con discapacidad $(n=206)^{2}$ señalaron: tener familiares con discapacidad $(\mathrm{n}=68)$, tener experiencia profesional con personas con discapacidad $(n=112)$, contar con experiencia en voluntariado con personas con discapacidad $(n=21)$ y tener amigos o conocidos $(n=55)$. Los participantes refieren haber tenido experiencia con personas con discapacidad física $(n=62)$, sensorial $(n=38)$, intelectual (n=96) y múltiple $(\mathrm{n}=14)$.

$\overline{2}$ En este estudio los participantes afirman haber tenido contacto con más de un tipo de personas con discapacidad de distintas formas: amigos y familiares, experiencia profesional y conocidos, etc. 
Respecto a las actitudes hacia las personas con discapacidad, según la escala de Verdugo, Arias y Jenaro (1994), la puntuación promedio de los ítems para esta muestra es de 4,8 (DT = 0,57), lo cual es indicativo de una actitud favorable hacia las personas con discapacidad. El análisis descriptivo de cada uno de los factores indica valores positivos (Tabla 4).

Tabla 4 - Estadísticos descriptivos de la escala total y subescalas.

\begin{tabular}{l|c|c|c|c|c|c|c}
\hline \multirow{2}{*}{ Variables } & \multirow{2}{*}{ Media } & \multirow{2}{*}{$\begin{array}{c}\text { Media } \\
\text { teórica }\end{array}$} & DT & \multicolumn{2}{|c|}{ Rango actual } & \multicolumn{2}{c}{ Rango posible } \\
\cline { 5 - 8 } & & & Min. & Max. & Min. & Max. \\
\hline Actitudes-global & 178,8 & - & 21,3 & 69 & 220 & 37 & 222 \\
\hline Factor 1 & 44,9 & 45,75 & 6,9 & 21 & 60 & 10 & 60 \\
\hline Factor 2 & $1.155,3$ & 56,69 & 7,9 & 17 & 66 & 11 & 66 \\
\hline Factor 3 & 38 & 30,52 & 4,8 & 7 & 42 & 7 & 42 \\
\hline Factor 4 & 522,5 & 21,56 & 3,6 & 11 & 30 & 5 & 30 \\
\hline Factor 5 & 18 & 16,79 & 3,6 & 7 & 24 & 4 & 24 \\
\hline
\end{tabular}

DT: Desviación Típica.

Fuente: Base de datos de la investigación.

Elaboración de las autoras.

Para los factores 1 (Valoración de capacidades y limitaciones) y 2 (Reconocimiento/ Negación de derechos), las medias de la muestra se sitúan ligeramente por debajo de lo esperado teóricamente; y para los factores 3 (Implicación personal), 4 (Calificación genérica) y 5 (Asunción de roles) son superiores.

Las pruebas realizadas para la comparación entre grupos (Prueba T de Student para dos muestras independientes, y ANOVA de un factor, Bonferroni para muestras múltiples) indican que no existen diferencias significativas en función del sexo, edad, experiencia de contacto con personas con discapacidad, razón del contacto o frecuencia del contacto. Al ser este un factor especialmente relevante, examinaremos con mayor detenimiento las diferencias en función del contacto previo con la discapacidad.

En ese sentido, a pesar de no haber diferencias significativas, las puntuaciones medias de las personas que señalan tener o haber tenido relación con personas con discapacidad son superiores en todos los factores, que aquellas que no. Además, se encuentran diferencias significativas en los ítems siguientes: las personas con discapacidad deberían tener las mismas oportunidades para el empleo que los demás $(\mathrm{t}=2.590$; $\mathrm{gl}=306 ; \mathrm{p}=0,010)$; muchas personas con discapacidad pueden ser profesionales competentes $(\mathrm{t}=2.284 ; \mathrm{gl}=306 ; \mathrm{p}=0,023)$; a las personas con discapacidad se les debería impedir votar $(\mathrm{t}=2.244 ; \mathrm{gl}=306 ; \mathrm{p}=0,026)$; las personas con discapacidad pueden hacer muchas cosas tan bien como cualquier otra persona $(\mathrm{t}=2.816 ; \mathrm{gl}=306$; $\mathrm{p}=0,005)$; la mayoría de las personas con discapacidad sienten que son tan valiosas 
como cualquiera $(\mathrm{t}=2.550 ; \mathrm{gl}=306 ; \mathrm{p}=0,011)$; se debería prevenir que las personas con discapacidad tuvieran hijos ( $\mathrm{t}=2.460 ; \mathrm{gl}=306 ; \mathrm{p}=0,014)$.

En todos estos casos, las personas que han tenido contacto con la discapacidad refieren actitudes más positivas. En cuanto a la diferencia en función del tipo de discapacidad con el que se ha mantenido contacto previo, únicamente existen diferencias significativas en el factor 3 (Implicación personal) entre aquellos participantes que han tenido relación con personas con discapacidad física y aquellos que han tenido relación con personas con discapacidad sensorial (T-test corregido por Bonferroni, $\mathrm{p}=0,004)$, mostrando los primeros actitudes más positivas.

\section{DISCUSIÓN}

La universidad como contexto educativo se encuentra en México aún lejos de considerarse un entorno plenamente inclusivo. En ese sentido, son varios los autores que hablan de cierta resistencia al cambio en el sistema universitario (Cruz y Casillas, 2017; Tapia y Manosalva, 2012). La propia estructura organizativa, las barreras arquitectónicas, la falta de servicios que promuevan la inclusión o las actitudes negativas hacia la discapacidad pueden ser factores explicativos de dicha resistencia. Si bien es complejo analizar toda esta red de influencias, parece que las actitudes acerca de las personas con discapacidad ejercerían un rol importante, como barreras a la plena inclusión. En ese sentido, la investigación durante los últimos años apuesta por el análisis de dichas variables, tanto en contextos universitarios como en otros niveles educativos.

Teniendo en cuenta dicho marco, se han presentado en este trabajo dos estudios en un contexto universitario, con muestras de estudiantes de posgrado, acerca de sus prejuicios y actitudes hacia las personas con discapacidad. Se han utilizado para ello dos instrumentos, con el objetivo adicional de testar su funcionamiento en México, dada la falta de herramientas específicas que se ajusten al contexto cultural y social de este país. Las respuestas de los participantes en ambos estudios reflejan, mayoritariamente, una posición favorable hacia las personas con discapacidad (escasos prejuicios y actitudes positivas). La tendencia de los universitarios a mantener actitudes positivas ha sido señalada por investigaciones previas como las de Araya-Cortés, González-Arias y Cerpa-Reyes (2014), Moreno et al. (2006), Upton y Harper (2002), Polo, Fernández y Díaz (2011) o Gómez y Infante (2004), considerando a este grupo como una población proinclusión respecto a otros colectivos. Investigaciones con muestras de diferentes edades y background cultural y social evidencian actitudes menos positivas (Jiménez y Huete, 2002).

En el Estudio 1 se ha utilizado la escala sobre actitudes explícitas e implícitas de Akrami et al. (2006). Es, desde nuestro conocimiento, la primera vez que se utiliza esta escala en el contexto de este país, por lo que supone un trabajo novedoso. Sin embargo, los datos arrojados por los análisis no son del todo satisfactorios. En primer lugar, para esta muestra los datos sobre la fiabilidad de las dimensiones de la escala no son aceptables, por lo que se optó por realizar los análisis de la escala en su conjunto. En ese sentido, este instrumento no nos permite discriminar los dos tipos de prejuicios. Si bien la presencia de prejuicios, clásicos y modernos, parece 
ser común en diferentes contextos y momentos históricos (Akrami et al., 2006), podemos inferir que el contenido de los mismos es probable que varíe en función del entorno socio-cultural. No en vano, recientemente, la investigación se ha interesado por otro tipo de factores que pudieran estar ejerciendo influencia en las actitudes hacia la discapacidad, relacionados con valores culturales (Hampton y Xiao, 2007; Scior, 2011; Scior et al., 2010).

En este caso, entendemos que la realidad de la muestra mexicana, determinada por condiciones económicas, históricas, sociales y políticas concretas, es la que podría explicar cuáles son los prejuicios hacia la discapacidad, y cómo varían a lo largo del tiempo. Algunas de esas condiciones pueden estar relacionadas con aquellas que se viven en el resto del país, por ejemplo, la estrecha relación entre condiciones de pobreza y población con discapacidad, la fragmentación cultural, la brecha económica entre ciertos grupos de población o los bajos índices de educación inclusiva que no promueven el contacto entre personas con discapacidad y el resto de la población (Blanco, 2006).

En segundo lugar, no se han encontrado diferencias significativas entre los grupos, de modo que las variables incluidas no parecen ejercer influencia sobre las actitudes o bien se encuentran asociadas a muchas otras variables no contempladas en nuestra investigación. Las propias características de la muestra, quizá demasiado homogénea, probablemente hayan influido en dichos resultados. No obstante, algunos otros trabajos coinciden en señalar la ausencia de diferencias entre grupos (Bausela, 2009; García y Hernández, 2011). A pesar de ello, se encuentran diferencias menores en algunos de los ítems en función de determinadas variables. Se corrobora que las mujeres y los jóvenes mantienen actitudes más positivas hacia las personas con discapacidad (Domínguez et al., 2014; García y Hernández, 2011; Martínez y Bilbao, 2011).

En cuanto al Estudio 2, se utilizó la Escala Actitudes hacia las personas con discapacidad, Forma G, de Verdugo, Arias y Jenaro (1994), una herramienta ampliamente aplicada en contextos diversos, durante años, para la valoración de actitudes. La muestra participante manifiesta actitudes positivas hacia las personas con discapacidad, como revelan las puntuaciones medias cercanas a la media tanto en la escala completa como en cada factor analizado. Este hallazgo es compartido por investigaciones que han utilizado el mismo instrumento, como las de Araya-Cortés, González-Arias y Cerpa-Reyes (2014), Gómez e Infante (2004), Moreno et al. (2006), Martínez y Bilbao (2011) o Polo y López (2006). Las valoraciones más positivas las encontramos en el factor 3 (Implicación personal), lo cual es indicativo de una buena disposición ante la interacción con personas con discapacidad en ámbitos personales, sociales y laborales. Estudios como los de Araya-Cortés, González-Arias y Cerpa-Reyes (2014), Martínez y Bilbao (2011) coinciden en señalar este factor como el más positivo; mientras que otros como Moreno et al. (2006) o García y Hernández (2011) precisamente lo señalan como el factor menos valorado. En nuestro trabajo, existen tres factores cuyas puntuaciones promedio está por debajo de la media esperada teóricamente: Valoración de capacidades y limitaciones y Reconocimiento/Negación de derechos. Por lo tanto, las actitudes más negativas de la muestra están referidas a la valoración sobre las personas respecto 
su capacidad de aprendizaje (aspecto especialmente relevante dado el ámbito universitario al que nos referimos) y al reconocimiento de sus derechos fundamentales (por ejemplo, derecho al voto).

Por otra parte, no se han encontrado diferencias reseñables en las puntuaciones de cada factor, en función del sexo, la edad, la experiencia de contacto previo con personas con discapacidad, la razón de dicho contacto o la frecuencia. Si bien algunos estudios refieren diferencias entre grupos, como se ha señalado previamente, también existe un buen número de investigaciones que refieren no encontrar diferencias significativas en función de variables como edad, sexo o titulación, en el caso de universitarios (Bausela, 2009; García y Hernández, 2011; Gómez y Infante, 2004). Mención aparte hemos realizado de la variable Contacto, cuyos hallazgos al respecto resultan contradictorios. En el presente estudio no se han encontrado diferencias significativas en función del contacto de manera similar a trabajos como el de Araya-Cortés, González-Arias y Cerpa-Reyes (2014). Por su parte, Bausela (2009) o Polo, Fernández y Díaz. (2011) sí señalan diferencias en determinados factores (mejores actitudes en aquellos que han tenido relación), si bien la razón o la frecuencia no parecen ejercer influencia. Por el contrario, Domínguez et al. (2014) encuentra una correlación entre frecuencia de contacto y actitudes: a mayor contacto con personas con discapacidad, mejores actitudes, en la puntuación global de la escala.

En el caso de nuestro estudio, a pesar de no hallar diferencias significativas, las puntuaciones medias tienden a ser más altas en aquellas personas que han tenido relación con la discapacidad, tal y como ocurre en otros trabajos (Martínez y Bilbao, 2011). La cuestión de si el contacto previo con las personas con discapacidad, por sí mismo, genera actitudes favorables ha sido abordada por algunos autores en profundidad. Parece ser, en ese sentido, que es más importante la calidad y el tipo de relación que la cantidad o el mero conocimiento de personas con discapacidad (MacManus, Feyes y Saucier, 2010), dato especialmente importante de cara a la planificación de acciones de sensibilización y erradicación de actitudes negativas o prejuicios. Otros estudios (Parasuram, 2006), curiosamente, encuentran actitudes más negativas en personas que han tenido contacto previo con la discapacidad, quizá atribuibles al cansancio de una relación de cuidado o atención más intensa. Precisamos de trabajos futuros para ahondar en estas variables cuyo análisis vemos que es complejo.

Cabe señalar algunas limitaciones de este trabajo con el fin de interpretar adecuadamente los datos expuestos. En primer lugar, la escasa representatividad de la muestra respecto a la población general universitaria que no permite generalizar conclusiones (todos los participantes están cursando estudios de maestría en el área de pedagogía, habiendo realizado un muestreo intencional). Quedaría por analizar el contenido específico acerca de la discapacidad que puede formar parte de dichos estudios así como el tipo de formación previa recibida. En segundo lugar, el alcance relativo de los instrumentos que, en el caso del Estudio 1, no presenta índices de fiabilidad aceptables en cuanto a las subescalas. En ese sentido, la escala de Verdugo, Arias y Jenaro (1994) se presenta como más adecuada para la valoración de las actitudes. Aún con ello, el uso de escalas autoadministradas — aunque muy difundido — ha 
sido cuestionado por muchos en cuanto a su incapacidad para predecir la conducta real de forma consistente. Así pues, cabría preguntarse por la validez de las herramientas utilizadas para la valoración de actitudes que, siendo mayoritariamente positivas en muchas investigaciones, no tienen por qué tener asociados mayores índices de inclusión. Es decir, ¿en qué medida es "real" la conexión entre las actitudes mostradas y la conducta respecto a las personas con discapacidad? Consideramos relevante seguir profundizando en el uso y adecuación de instrumentos como el de Akrami et al. (2006) que pueden aportar luz en esta cuestión. En tercer lugar, se ha realizado un análisis descriptivo y comparativo que permite identificar cierta tendencia en cuanto a las actitudes de los diferentes grupos de universitarios, pero impide hablar de causalidad.

\section{CONCLUSIONES}

Los datos aportados en este trabajo, en líneas generales, señalan una actitud positiva hacia las personas con discapacidad por parte de estudiantes universitarios: valoran sus capacidades, son conscientes de la importancia de defender los derechos de este grupo de población, reconocen su valía personal y no asumen estereotipos centrados en ideas como la excesiva dependencia, la victimización o el trato de favor. En ese sentido, la tendencia a valorar de forma realista y positiva a las personas con discapacidad es una buena señal de cara a la eliminación de barreras intangibles (prejuicios y actitudes negativas), por lo que entendemos que al menos la cultura universitaria, en cuanto a actitudes concretas sobre esta realidad, es favorable.

No obstante, y dadas las limitaciones señaladas previamente, los resultados deben ser interpretados con cautela. En ese sentido, urge ampliar los estudios sobre actitudes con muestras más amplias y heterogéneas. Los futuros trabajos deberían orientarse a desarrollar instrumentos válidos y adaptados a la realidad mexicana, incluir un amplio rango de variables que expliquen la variabilidad de perspectivas de la población acerca de las personas con discapacidad y diseñar acciones para la superación de prejuicios y que favorezcan, de este modo, el respeto y reconocimiento de la diversidad como parte de la sociedad. Sería interesante profundizar en la perspectiva acerca de prejuicios explícitos e implícitos, ateniendo a la idiosincrasia propia de dicho país. Quizá el desarrollar instrumentos culturalmente adaptados nos permitiera una comprensión más completa de las actitudes y prejuicios presentes. En ese sentido, puede que estudios como los presentados sean demasiado genéricos y no lleguen a abordar actitudes negativas enraizadas en la población mexicana, más allá de actitudes generales.

Por otra parte, la presencia de una actitud favorable no nos permite establecer conclusiones definitivas ni realizar predicciones acerca del comportamiento de los universitarios para con personas con discapacidad (por ejemplo, compañeros). La escasa presencia de personas con discapacidad en la universidad (como alumnos/as o profesionales) hace difícil estudiar la relación verdadera entre actitudes y conductas; más aún cuando la investigación advierte de que el mero contacto, por sí mismo, no asegura una buena disposición o interacción. En ese sentido, sería adecuado desarrollar acciones que promovieran verdaderos intercambios entre personas, de igual a igual, no basados en relaciones de compasión o caridad, como ha ocurrido durante décadas 
anteriores. La verdadera inclusión llegará en el momento en que todas las personas, diversas en sus capacidades, orígenes, circunstancias y motivaciones, compartan espacios educativos en igualdad de condiciones. Y hasta ese momento, la investigación debe seguir profundizando en las causas de la exclusión para poder avanzar.

\section{REFERENCIAS}

AKRAMI, N.; EKEHAMMAR, B.; ARAYA, T. Classical and modern racial prejudice: a study of attitudes toward immigrants in Sweden. European Journal of Social Psychology, Muenster, v. 30, n. 4, p. 521-532, 2000. https://doi.org/10.1002/1099-0992(200007/08)30:4<521::AID-EJSP5>3.0.CO;2-N AKRAMI, N.; EKEHAMMAR, B.; CLASESSON, M.; SONNANDER, K. Classical and modern prejudice: attitudes toward people with intellectual disabilities. Research in Developmental Disabilities, London, v. 27, n. 6, p. 605-617, 2006. https://doi.org/ 10.1016/j.ridd.2005.07.003

ALCÁNTARA, A.; NAVARRETE, Z. Inclusión, equidad y cohesión social en las políticas de educación superior en México. Revista Mexicana de Investigación Educativa, México, DF, v. 19, n. 60, p. 213-239, 2014.

ALLPORT, G. The nature of prejudice. Cambridge: Addison Wesley, 1954.

ARAYA-CORTÉS, A.; GONZÁLEZ-ARIAS, M.; CERPA-REYES, C. Actitud de universitarios hacia las personas con discapacidad. Educación y Educadores, Chía, v. 17, n. 2, p. 289-305, 2014. https://doi.org/10.5294/edu.2014.17.2.5

BARR,J.; BRACCHITTA, K. Attitudes toward individuals with disabilities: the effects of contact with different disability types. Current Psychology, New York, v. 34, n. 2, p. 223-238, 2015. https://doi.org/10.1007/s12144-014-9253-2

BARRADAS, M.; ROBLEDO, M.; GUZMÁN, M.; GUTIÉRREZ, L.; SÁNCHEZ, J. Actitudes hacia las personas con discapacidad en académicos de la Universidad Veracruzana. Revista Iberoamericana de Producción Académicay Gestión Educativa, México, v. 1, n. 2, s/p., 2014.

BAUSELA, E. Actitudes hacia la discapacidad: estudio de algunas propiedades psicométricas en una muestra de universitarios mexicanos. Revista Iberoamericana de Educación, Madrid, v. 49, n. 6, p. 1-10, 2009.

BLANCO, R. La equidad y la inclusión social: uno de los desafíos de la educación y la escuela hoy. Revista Iberoamericana sobre Calidad, Eficacia y Cambio en Educación, Madrid, v. 4, n. 3, p. 1-15, 2006.

CAIRNS, B.; MCCLATCHEY, K. Comparing children's attitudes towards disability. British Journal of Special Education, Austin, v. 4, n. 3, p. 124-129, 2013. https://doi.org/10.1111/1467-8578.12033

CRUZ, R. Discapacidad y educación superior: ¿una cuestión de derechos o buenas voluntades? CPU-e Revista de Investigación Educativa, v. 23, p. 1-23, jul./dic. 2016. Disponible en: http://www.scielo.org.mx/pdf/cpue/n23/1870-5308-cpue-23-00002.pdf. Acceso: 1 oct. 2018. 
CRUZ, R.; CASILLAS, M.A. Las instituciones de educación superior y los estudiantes con discapacidad en México. Revista de la Educación Superior, México, v. 46, n. 181, p. 37-53, 2017. https://doi.org/10.1016/j.resu.2016.11.002

DACHEZ,J.; NDOBO, A.; AMELINE, A. French validation of the Multidimensional Attitude Scale towards persons with disabilities (MAS): the case of attitudes toward autism and heir moderating factors. Journal of Autism and Developmental Disorders, Conneticut, v. 45, n. 8, p. 2.508-2.518,2015.https://doi.org/10.1007/s10803-015-2417-6 DAVIS, J.; WATSON, N. Where are the children's experiences? Analysing social and cultural exclusion in "special” and "mainstream" schools'. Disability \& Society, London, v. 16, n. 5, p. 671-87, 2001. https://doi.org/10.1080/09687590120070060

DOMÍNGUEZ, S.; VILLEGAS, G.; BOCANEGRA, K.; AQUIJE, D.; CASTILLO, A.; DULANTO, N.; LLONTOP, C.; RIMACHI, M. Actitudes hacia las personas con discapacidad en una muestra de estudiantes universitarios de Lima Metropolitana. Revista Peruana de Psicología y Trabajo Social, Lima, v. 3, n. 1, p. 37-60, 2014.

EZCURRA, M. Resultados del informe final de la investigación: la calidad de la atención educativa de los niños y jóvenes con discapacidad de los centros de atención múltiple. México: Secretaría de Educación Pública, 2003.

FINDLER, L.; VILCHINSKY, N.; WERNER, S. The Multidimensional Attitudes Scale toward persons with disabilities (MAS): construction and validation. Rehabilitation Counseling Bulletin, Austin, v. 50, n. 3, p. 166-176, 2007. https://doi.org/10.1177/00343552070500030401

GARCÍA, G.; HERNÁNDEZ, S. Actitudes hacia la discapacidad de jóvenes y adultos de Chiapas. Universitas Psychologica, Bogotá, v. 10, n. 3, p. 817-827, 2011. https://doi.org/10.11144/Javeriana.upsy10-3.adja

GARCÍA, I. Educación inclusiva en Latinoamérica y el Caribe. El caso mexicano. Potosí: Editorial Universitaria Potosina, 2011.

GÓMEZ,V.; INFANTE,M.Actitudes de los estudiantes de educación hacia la integración de personas con discapacidad y hacia la educación multicultural. Cultura y Educación, London, v. 16, n. 4, p. 371-383, 2004. https://doi.org/10.1174/1135640042802455

GORECZNY, A.; BENDER, E.; CARUSO, G.; FEINSTEIN, C. Attitudes toward individuals with disabilities: results of a recent survey and implications of those results. Research in Developmental Disabilities: A MultidisciplinaryJournal, London, v. 32, n. 5, p. 1.596-1609, 2011. https://doi.org/10.1016/j.ridd.2011.02.005

HAMPTON, N.; XIAO, F. Attitudes toward people with developmental disabilities in Chinese and American students: the role of cultural values, contact, and knowledge. Journal of Rehabilitation, Washington, v. 7, n. 4, p. 23-32, 2007.

HARPER, D.; PETERSON, D. Children of the Philippines: attitudes toward visible physical impairment. Cleft Palate-Craniofacial Journal, Chapel Hill, v. 38, n. 6, p. 566-576,2001.https://doi.org/10.1597/1545-1569_2001_038_0566_cotpat_2.0.co_2 INEGI - Instituto Nacional de Estadística y Geografía. Encuesta nacional sobre discriminación en México - ENADIS. Ciudad de México: INEGI, 2005. 
INEGI - Instituto Nacional de Estadística y Geografía. Encuesta nacional sobre discriminación en México - ENADIS. Ciudad de México: INEGI, 2010.

INEGI - Instituto Nacional de Estadística y Geografía. Encuesta nacional sobre discriminación en México - ENADIS. Ciudad de México: INEGI, 2017.

JIMÉNEZ, A.; HUETE, A. La discriminación por motivos de discapacidad. Análisis de las respuestas recibidas al cuestionario sobre discriminación por motivos de discapacidad promovido por el CERMI Estatal. Madrid: CERMI, 2002.

MACMANUS, J.; FEYES, K.; SAUCIER, D. Contact and knowledge as predictors of attitudes toward individuals with intellectual disabilities. Journal of Social and Personal Relationships, Toronto, v. 28, n. 5, p. 579-590, 2010. https://doi.org/10.1177/0265407510385494

MARTÍNEZ, M. A.; BILBAO, M. C. Los docentes de la Universidad de Burgos y su actitud hacia las personas con discapacidad. Revista Española sobre la Discapacidad Intelectual Siglo Cero, Madrid, v. 42, n. 4, p. 50-78, 2011.

MÉXICO. Ley General para la Inclusión de las Personas con Discapacidad. Diario Oficial de la Federación, 2011. [Última reforma publicada 12 jul. 2018]. Disponible en: http://www.diputados.gob.mx/LeyesBiblio/pdf/LGIPD_120718.pdf.Acceso: 1 oct. 2018. MORENO, F.J.; RODRÍGUEZ, I.; SALDAÑA, D.; AGUILERA, A. Actitudes ante la discapacidad en el alumnado universitario matriculado en materias afines. Revista Iberoamericana de Educación, Madrid, n. 40, p. 1-12, 2006.

MORIN, D.; RIVARD, M.; CROCKER, A.; CARON, J. Public attitudes towards intellectual disability: a multidimensional perspective. Journal of Intellectual Disability Research, United Kingdom, v. 57,n.3, p. 279-292,2013. https://doi.org/10.1111/jir.12008 OUELLETTE-KUNTZ, H.; BURGE, P.; BROWN, H.; ARSENAULT, E. Public attitudes towards individuals with intellectual disabilities as measured by the concept of social distance. Journal of Applied Research in Intellectual Disabilities, London, v. 23, n. 2, p. 132-142, 2010. https://doi.org/10.1111/j.1468-3148.2009.00514.x PALACIOS, A.; BARIFFI, F. La discapacidad como una cuestión de derechos humanos. Madrid: Cinca, 2007.

PARASURAM, K. Variables that affect teachers' attitudes towards disability and inclusive education in Mumbai, India. Disability \& Society, London, v. 21, n. 3, p. 231-242, 2006. https://doi.org/10.1080/09687590600617352

PETTIGREW, T.; TROPP, L. A meta-analytic test of intergroup contact theory. Journal of Personality and Social Psychology, Washington, v. 90, n. 5, p. 751-783, 2006. https://doi.org/10.1037/0022-3514.90.5.751

POLO, M. T.; LÓPEZ, M. D. Actitudes hacia las personas con discapacidad de estudiantes de la Universidad de Granada. Revista Española de Orientación y Psicopedagogía, Madrid, v. 17, n. 2, p. 195-211, 2006. https://doi.org/10.5944/reop.vol.17.num.2.2006.11346

POLO, T.; FERNÁNDEZ, C.; DÍAZ, C. Estudio de las actitudes de estudiantes de ciencias sociales y psicología: relevancia de la información y contacto con personas discapacitadas. Universitas Psychologica, Bogotá, v. 10, n. 1, p. 113-123, 2011. 
ROMERO, S.; GARCÍA, I. Educación especial en México. Desafíos de la educación inclusiva. Revista Latinoamericana de Educación Inclusiva, Madrid, v. 7, n. 2, p. 77-91, 2013.

SCIOR, K. Public awareness, attitudes and beliefs regarding intellectual disability: a systematic review. Research in Developmental Disabilities, London, v. 32, n. 6, p. 2.164-2.182, 2011. https://doi.org/10.1016/j.ridd.2011.07.005

SCIOR, K.; FURNHAM, A. Development and validation of the intellectual disability literacy scale for assessment of knowledge, beliefs and attitudes to intellectual disability. Research in Developmental Disabilities, London, v. 32, n. 5, p. 1.530-1.541, 2011. https://doi.org/10.1016/j.ridd.2011.01.044

SCIOR, K.; KAN, K.; MCLOUGHLIN, A.; SHERIDAN, J. Public attitudes toward people with intellectual disabilities: a cross-cultural study. Intellectual and Developmental Disabilities, Washington, v. 48, n. 4, p. 278-289, 2010. https://doi.org/10.1352/1934-9556-48.4.278

SEO, W.; CHEN, R. Attitudes of college students toward people with disabilities. Journal of Applied Rehabilitation Counseling, Alexandria, v. 40, n. 4, p. 3-8, 2009.

TAPIA, C.; MANOSALVA, S. Inclusión de estudiantes con discapacidad en la educación superior. Revista de Estudios y Experiencias en Educación, Concepción, v. 11, n. 22, p. 13-34, 2012.

TEN-KLOOSTER, P.; DANNENBERG, J.; TAAL, E.; RASKER, J. Attitudes towards people with physical or intellectual disabilities: nursing students and nonnursing peers. Journal of Advanced Nursing, Hoboken, v. 65, n. 12, p. 2.562-2.573, 2009. https://doi.org/10.1111/j.1365-2648.2009.05146.x

TRIANDIS, H. Attitude and attitude change. New York: Wiley, 1971.

UNESCO - Organización de las Naciones Unidas para la Educación, la Ciencia y la Cultura. Educación para todos en América Latina: un objetivo a nuestro alcance. Informe regional de monitoreo de educación para todos 2003. Santiago de Chile: UNESCO, 2004.

UNESCO - Organización de las Naciones Unidas para la Educación, la Ciencia y la Cultura. Panorama educativo 2005: progresando hacia las metas. Informe preliminar del proyecto regional de indicadores educativos de la cumbre de las Américas. Santiago de Chile: UNESCO; SEP, 2005.

UPTON, T.; HARPER, D. Multidimensional Disability Attitudes and Equitable Evaluation of Educational Accommodations by college students without disabilities.Journal of Postsecondary Education and Disability, Huntersville, v. 15, n. 2, p. 115-130, 2002.

VERDUGO, M. A. La concepción de discapacidad en los modelos sociales. Salamanca: Amarú, 2001.

VERDUGO, M. A.; ARIAS, B.; JENARO, C. Actitudes hacia las personas con minusvalía. Madrid: Ministerio de Asuntos Sociales; Instituto Nacional de Servicios Sociales, 1994.

VERDUGO, M. A.; SCHALOCK, R. L. Discapacidad e inclusión: manual para la docencia. Salamanca: Amarú, 2013. 
WERNER, S.; CORRIGAN, P.; DITCHMAN, N.; SOKOL, K. Stigma and intellectual disability: a review of measures and future directions. Research in Developmental Disabilities, London, v. 33, n. 2, p. 748-765, 2012. https://doi.org/10.1016/j.ridd.2011.10.009

\section{SOBRE LAS AUTORAS}

Araceli Arellano Torres es doctora en psicopedagogía por la Universidad de Navarra (España). Profesora de la misma institución. E-mail: aarellanot@unav.es

Martha Leticia Gaeta González es doctora en psicología y aprendizaje por la Universidad de Zaragoza (España). Profesora de la Universidad Popular Autónoma del Estado de Puebla (México).

E-mail: marthaleticia.gaeta@upaep.mx

Feli Peralta López es doctora en ciencias de la educación por la Universidad de Navarra (España). Profesora de la misma institución. E-mail:fperalta@unav.es

Judith Cavazos Arroyo es doctora en dirección y marketing por la Universidad Popular Autónoma del Estado de Puebla (México). Profesora de la misma institución.

E-mail: fudith.cavazos@upaep.mx

Recibido el 07 de noviembre de 2018 Aprobado el 25 de febrero de 2019 